\title{
Comparaison entre trois méthodes de mesure directe de fortes concentrations en radon dans l'air
}

\author{
A. RENOUX (*), A. MOUDEN $\left({ }^{*}\right)$, G. MADELAINE $\left({ }^{* *}\right)$
}

(Manuscrit reşu le 5 mars 1975)

\begin{abstract}
RÉSUMÉ
On étudie et on compare trois méthodes de mesure directe du radon dans le cas d'atmosphères fortement radioactives. La première est la méthode des fioles scintillantes du CEA-STEPPA, qui servira de référence. La seconde est la méthode dite des deux filtres, utilisant une chambre de désintégration parcourue par un courant d'air de débit connu. La dernière utilise le piégeage électrostatique du dépôt actif du radon à l'aide d'une haute tension continue négative. Ces trois procédés ont été utilisés dans une mine d'uranium, et donnent des résultats comparables. On traite, enfin, de leurs avantages respectifs et de leur sensibilité.
\end{abstract}

\begin{abstract}
A comparison of three methods of direct measurement of high concentrations of atmospheric radon.

Three methods of direct measurement of radon in the case of highly radioactive atmospheres were studied and compared. The first one used the CEA-STEPPA scintillating bottles as standards. In the second technique, the "double filter" system, a known air flow rate was drawn through a decay chamber. In the last technique, the radon radioactive deposit was trapped electrostatically by means of a negative direct-current high voltage. All the three methods were operated in a uranium mine and gave similar results. Their respective advantages and sensitivities were tested.
\end{abstract}

(*) Laboratoire de Physique des Aérosols et de Radioactivité atmosphérique. Faculté des Sciences de Brest (U.B.O.), avenue Victor-Le-Gorgeu, 29200 Brest.

(**) Service technique d'Études de Protection et de Pollution atmosphérique du C.E.A., Département de Protection, B.P. $n^{\circ}$ 6, 92260 Fontenay-aux-Roses. 
Nous nous proposons de comparer trois méthodes de mesure directe du radon, dans le cas d'activités élevées rencontrées dans des mines d'uranium ou dans des atmosphères artificiellement enrichies en radon. Il existe, on le sait, de très nombreux procédés, et nous avons retenu trois d'entre eux nous paraissant les plus commodes d'emploi.

\section{LA MÉTHODE DES FIOLES SCINTILLANTES}

Cette technique, mise au point par Pradel et BILlard [1] est suffisamment connue pour que nous ne nous attardions pas à la décrire en détail. Rappelons qu'il s'agit de fioles de $125 \mathrm{~cm}^{3}$, tapissées intérieurement d'une couche de sulfure de zinc activé à l'argent. On la vide au préalable, et l'air chargé en radon est introduit à l'aide d'une aiguille hypodermique. La mesure s'effectue $3 \mathrm{~h}$ plus tard (afin d'avoir l'équilibre entre le radon et son dépôt actif), en plaçant la fiole sur un photomultiplicateur (comptages $\alpha$ ). $100 \mathrm{pCi} / 1\left(10^{-10} \mathrm{Ci} / \mathrm{l}\right)$ correspondent à 50 impulsions par minute. La sensibilité est donc de l'ordre de $5 \mathrm{pCi} / \mathrm{l}$.

Cette méthode nous servira de référence, nous la comparerons aux deux autres.

\section{LA MÉTHODE DES DEUX FILTRES}

Le principe en est le suivant : on aspire l'air chargé en radon à travers deux filtres séparés par une chambre de désintégration; le filtre d'entrée arrête tous les aérosols et, en particulier, les descendants du radon. Le filtre de sortie retient le dépôt actif formé par désintégration du radon lors de sa traversée de la chambre. La concentration en radon se déduit de l'activité $\alpha$ du filtre de sortie. Cette méthode présente l'intérêt de n'exiger aucune connaissance préalable de l'état d'équilibre du radon et de ses descendants.

Le principe de la chambre de désintégration, et son application à la mesure automatique du radon, a été étudié par IsRAËL [2], FONTAN [3] puis, plus tard, par d'autres auteurs dont Newstern et al. [4], qui ont mis au point un système automatique dans lequel la chambre et la partie mesure sont solidaires, ce qui permet de suivre l'évolution du radon par déroulement du filtre devant un photomultiplicateur, mais nécessite une construction minutieuse de l'appareillage de mesure et un étalonnage de la chambre à partir d'une source connue en radon pour chaque variation de paramètre.

Thomas et Le Clare [5] ont exposé des calculs dont le but est de développer une équation donnant directement la concentration de radon à partir de données expérimentales utilisables : dimension de la chambre cylindrique, débit et temps de prélèvement $t$, activité $x$ du filtre comptée dans l'intervalle $T_{1}-T_{2}$ après l'arrêt du prélèvement. Cette dernière méthode est celle que nous avons retenue.

Dans le cas de fortes activités, il est possible d'utiliser une chambre de désintégration de volume assez faible et, de ce fait, très peu encombrante, par ailleurs, on peut modifier sans aucun inconvénient les différents paramètres : temps de prélèvement et de comptage, débit et, éventuellement, volume de la chambre de désintégration, sans aucun étalonnage préalable. 
a) CAlculs

Soit $V$ (en litres) le volume de la chambre cylindrique, $Q$ la concentration du radon en atomes/litre, $\lambda_{1}$ la constante de désintégration du radon $\left(\mathrm{min}^{-1}\right)$, $C_{\mathrm{Rn}}$ la concentration du radon en picocuries par litre.

$Q V \lambda_{1}$ est le nombre d'atomes de radium A formés par minute dans le tube.

Mais, seule une fraction $F_{f}$ de ce radium A arrive sur le filtre de sortie, l'autre partie diffusant sur les parois de l'enceinte. De ce fait, le nombre d'atomes arrivant sur le filtre de sortie par minute est $y=F_{f} Q V \lambda_{1}$.

La formation de radium $\mathbf{B}$ et $\mathbf{C}$ dans le tube est négligeable, du fait des faibles dimensions de ce dernier.

D'autre part,

$$
Q=2,22 \frac{C_{\mathrm{Rn}}}{\lambda_{1}} .
$$

D'où

$$
C_{\mathrm{Rn}}=\frac{0,45 y}{V_{f}} .
$$

Le coefficient $y$ n'est pas directement mesurable, mais il peut être relié au nombre $X$ de désintégrations $\alpha$ obtenues sur le filtre de sortie entre les instants $T_{1}$ et $T_{2}$.

On montre [5] que $y=X / Z E$, où $E$ est l'efficacité de comptage.

$Z$ est obtenu à partir de l'intégration des équations de type Bateman relatives à l'accumulation et à la décroissance des descendants du radon sur le filtre.

On aboutit à

$$
Z=4,504 A F+164,69 B G-93,31 C H,
$$

où $Z$ est fonction de $t$ (durée de prélèvement), $T_{1}, T_{2}$ et des constantes de désintégration $\lambda_{1}, \lambda_{2}, \lambda_{3}$ du radium $\mathrm{A}, \mathrm{B}$ et $\mathrm{C}$.

Les expressions $A, B, C$ et $F, G, H$ ainsi que quelques valeurs de $Z$ pour des valeurs choisies de $t, T_{1}$ et $T_{2}$ sont données dans le tableau I.

La fraction $F_{f}$ qui tient compte de la diffusion des atomes de radium $\mathrm{A}$ créés " en vol " dans une chambre de désintégration cylindrique, est une fonction de $\mu=\pi D L / q$ d'après les calculs de TAN [6]. $L$ est la longueur de la chambre, $q$ le débit d'air en $\mathrm{cm}^{3} \cdot \mathrm{s}^{-1}, D$ le coefficient de diffusion du radium $\mathrm{A}$ que l'on prend égal à $0,085 \mathrm{~cm}^{2} \cdot \mathrm{s}^{-1}$. Cette valeur donne de bons résultats dans un domaine d'hygrométrie supérieure à $20 \mathrm{p}$. cent, contrairement à ce que l'on obtiendrait avec celles couramment admises - de l'ordre de 0,05 à $0,06 \mathrm{~cm}^{2} \cdot \mathrm{s}^{-1}$ [7].

Ainsi

$$
C_{\mathrm{Rn}}=\frac{0,45 X}{E Z V_{f}}
$$

vol. $10-\mathrm{N}^{0} 1$ 


\section{b) Appareillage}

Les dimensions du tube (fig. 1) sont le résultat d'un compromis entre la sensibilité et le peu d'encombrement désirés. La chambre de longueur $L=51,6 \mathrm{~cm}$ et de diamètre $\Phi=3,4 \mathrm{~cm}$ a été testée à des débits et des temps de prélèvements différents avec des filtres Schneider-Poelman roses de très haute efficacité.

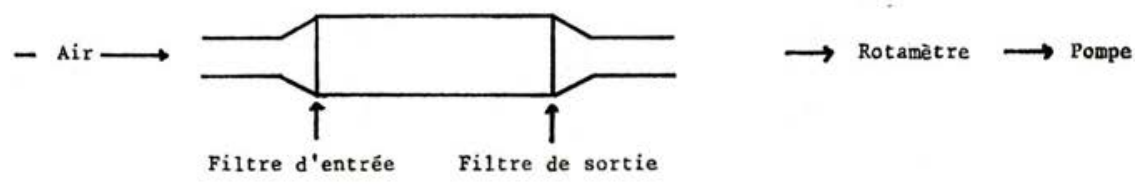

Fig. 1. - Méthode de Thomas.

Au cours de nos premiers essais comparatifs, nous avons obtenu, avec ce tube, des valeurs de $C_{\mathrm{Rn}}$ supérieures à celles mesurées avec les fioles scintillantes. Ceci était dû à une faible pénétration de l'aérosol radioactif dans la chambre, phénomène déjà observé par d'autres auteurs [8] avec un filtre à haute efficacité. L'utilisation d'un deuxième filtre $F_{2}$ à l'entrée, ramène la concentration calculée au taux normal.

Afin d'étudier plus en détail ce phénomène, nous avons comparé les activités $\alpha$ des deux filtres d'entrée (amont : $F_{1}$ et aval : $F_{2}$ ). Une série de mesures à $18 \mathrm{~cm} / \mathrm{s}$ a donné un rapport moyen d'activité : $F_{2} / F_{1}=6.10^{-3}$ légèrement supérieur à celui indiqué par BiLlaRD et al. [9], mais à des vitesses inférieures $(4 \mathrm{~cm} / \mathrm{s})$.

Le rendement du système de filtration à l'entrée du tube est défini par

$$
\mathbf{R}=\frac{\text { activité sur le filtre à essayer }}{\text { somme des activités sur les deux filtres }} \text {. }
$$

Un grand nombre de mesures effectuées sur plusieurs filtres roses a donné un rendement moyen de $R=99,4 \mathrm{p}$. cent pour la vitesse de $18 \mathrm{~cm} / \mathrm{s}$.

L'utilisation d'un troisième filtre d'entrée montre que, d'une part, le rendement des filtres $F_{1}+F_{2}$ est supérieur à $99,99 \mathrm{p}$. cent et que, d'autre part, ce troisième filtre ne modifie en rien l'activité du filtre de sortie.

Tenant compte de ces résultats, nous plaçons systématiquement deux filtres roses à l'entrée de notre tube.

De plus, les mesures doivent tenir compte des pertes de comptage dues à la pénétration des aérosols radioactifs dans le filtre de sortie (correction d'autoabsorption).

Cette pénétration a été calculée par comparaison avec le filtre millipore captant tout l'aérosol en surface [10], c'est-à-dire une efficacité $E=0,5$ sous $2 \pi$. 
Plusieurs essais ont donné les résultats suivants :

$E=0,44 \pm 0,02$ à une vitesse $9 \mathrm{~cm} / \mathrm{s}$.

$E=0,42 \pm 0,02$ à une vitesse $18 \mathrm{~cm} / \mathrm{s}$.

L'appareil a été testé par comparaison à des fioles scintillantes, en effectuant des prélèvements en circuit fermé, dans une enceinte contenant une forte concentration en radon. Un grand nombre de mesures à $10 \mathrm{l} / \mathrm{min}$ et à des temps de prélèvements variant de 1 à $5 \mathrm{~min}$, ont donné des résultats concordants et l'écart entre la moyenne de ces mesures et celles obtenues à l'aide des fioles scintillantes est de $4 \mathrm{p}$. cent.

\section{c) Sensibilité}

La sensibilité du tube dépend des conditions d'utilisation et, en particulier, du facteur $Z$.

Supposons que l'on fasse varier simplement le paramètre $t$ à un débit fixe de $10 \mathrm{l} / \mathrm{min}$ et à des temps de comptage de $5 \mathrm{~min}$ entre $T_{1}=1 \mathrm{~min}$ et $T_{2}=6 \mathrm{~min}$.

Pour notre tube, nous avons $E=0,42$.

La concentration en radon devient :

$$
C_{\mathrm{Rn}}=\frac{3,75 X}{Z},
$$

$X$ étant le nombre de coups comptés entre la première et la sixième minute.

$$
\begin{aligned}
& t=15 \text { min donne } C_{\mathrm{Rn}}=1,41 X ; \\
& t=10 \text { min donne } C_{\mathrm{Rn}}=1,62 X ; \\
& t=5 \text { min donne } C_{\mathrm{Rn}}=2,24 X ; \\
& t=1 \text { min donne } C_{\mathrm{Rn}}=7,60 X .
\end{aligned}
$$

Les relations ci-dessus montrent qu'un prélèvement de $15 \mathrm{~min}$, suivi d'une mesure à l'aide d'un scintillateur à faible bruit de fond autorisant l'exploitation d'un comptage de $X=7$ coups $/ 5 \mathrm{~min}$, permet de mesurer une concentration de $10 \mathrm{pCi} / 1$. Par contre, un prélèvement de $1 \mathrm{~min}$, toutes choses égales par ailleurs, ne permet pas de mesurer une concentration inférieure à $50 \mathrm{pCi} / 1$ avec le même pourcentage d'erreur. La sensibilité est divisée par un facteur 5 , montrant ainsi que la sensibilité diminue beaucoup avec le temps de prélèvement.

On s'aperçoit également que, à volume égal d'air prélevé, un débit plus faible donne une sensibilité meilleure. En effet, pour $V=101$ :

$$
\text { si } \left.\begin{array}{rl}
t & =10 \mathrm{~min} \\
q & =11 / \mathrm{min}
\end{array}\right\} \text { on a } \quad C_{\mathrm{Rn}}=5,15 X,
$$

alors que si $\left.\begin{array}{rl}t & =1 \mathrm{~min} \\ q & =10 \mathrm{l} / \mathrm{min}\end{array}\right\}$ on obtient $C_{\mathrm{Rn}}=7,6 X$.

vol. $10-\mathrm{N}^{\circ} 1$ 
Par ailleurs, la précision des résultats sera d'autant meilleure que le temps de comptage $T_{2}-T_{1}$ sera élevé, c'est-à-dire $Z$ grand pour un temps $t$ de prélèvement donné. Ceci est important dans le cas des faibles activités.

Notre tube de désintégration a servi à mesurer la concentration du radon dans les mines d'uranium de la Division minière de la Crouzille du C.E.A.

Pour un prélèvement de $10 \mathrm{~min}$ de durée et un comptage de $30 \mathrm{~min}$ $(Z=5,425)$, on a

$$
C=0,726 X
$$

On comptait, en moyenne, 220 coups, tout bruit de fond déduit, soit sensiblement une activité de l'ordre de $160 \mathrm{pCi} / 1$ en bon accord, là encore, avec la valeur mesurée à l'aide des fioles scintillantes.

Donc, ce type d'appareil permet de déterminer la concentration en radon de l'air d'une mine d'uranium.

Pour des concentrations plus faibles il est nécessaire d'augmenter le volume, et, par suite, les dimensions du tube. C'est ainsi qu'il a été construit une chambre de 9,441 de volume et de $1 \mathrm{~m}$ de longueur pouvant atteindre une sensibilité de $0,2 \mathrm{pCi} / 1$. Cette chambre peut être utilisée en mine mais ses dimensions constituent déjà une limite d'encombrement maximal. Il a été réalisé, par ailleurs, pour mesurer le radon atmosphérique, une chambre de 288 1, le débit de l'air étant de $2501 / \mathrm{min}$ [11].

\section{MESURE DU RADON PAR PIÉGEAGE DU DÉPÔT ACTIF}

\section{a) PRINCIPE}

L'air à étudier est introduit dans une chambre de désintégration, après passage au travers d'un filtre rose qui arrête les descendants du radon déjà formés. L'ensemble de la chambre de désintégration est monté sur un photomultiplicateur constitué par une sonde à grande surface, sensible aux rayons $\alpha$ et reliée à un ensemble de mesure et à un enregistreur.

Une fraction du radon se désintègre en radium $\mathrm{A}$ à l'arrivée dans la chambre. Ce radium A se trouve sous forme de petits ions positifs que l'on piège sur une plaque métallique portée à une tension négative. Le photomultiplicateur ne détectera que les $\alpha$ du radon qui atteignent le scintillateur placé sur lui, car le rayonnement $\alpha$ émis par désintégration du radium $\mathbf{A}$ capté par la plaque négative ne pourra être compté. En effet, la distance scintillateur-plaque métallique, de l'ordre de $8 \mathrm{~cm}$, est supérieure au parcours des rayons $\alpha$ dans l'air.

\section{b) Appareillage}

La chambre de désintégration est constituée par un cylindre en AFCODUR type C.E.N.F.A.R. de diamètre $\Phi=255 \mathrm{~mm}$ et de hauteur $h=8 \mathrm{~cm}$ (fig. 2). La partie supérieure est obturée par une plaque métal- 
lique $P$ reliée à une haute tension négative $-v$. A cette plaque, percée en son centre d'une ouverture permettant l'admission du radon, peut être adapté un filtre rose plaqué contre un disque de bronze fritté. Le système de mesure est un détecteur de type Nardeux doté d'une sonde $\alpha$ à grande surface (SA 125 DM 1) recouverte d'une pellicule de protection contre les rayons lumineux. Un enregistreur potentiométrique type Servotrace permet de suivre l'évolution du radon dans l'air.

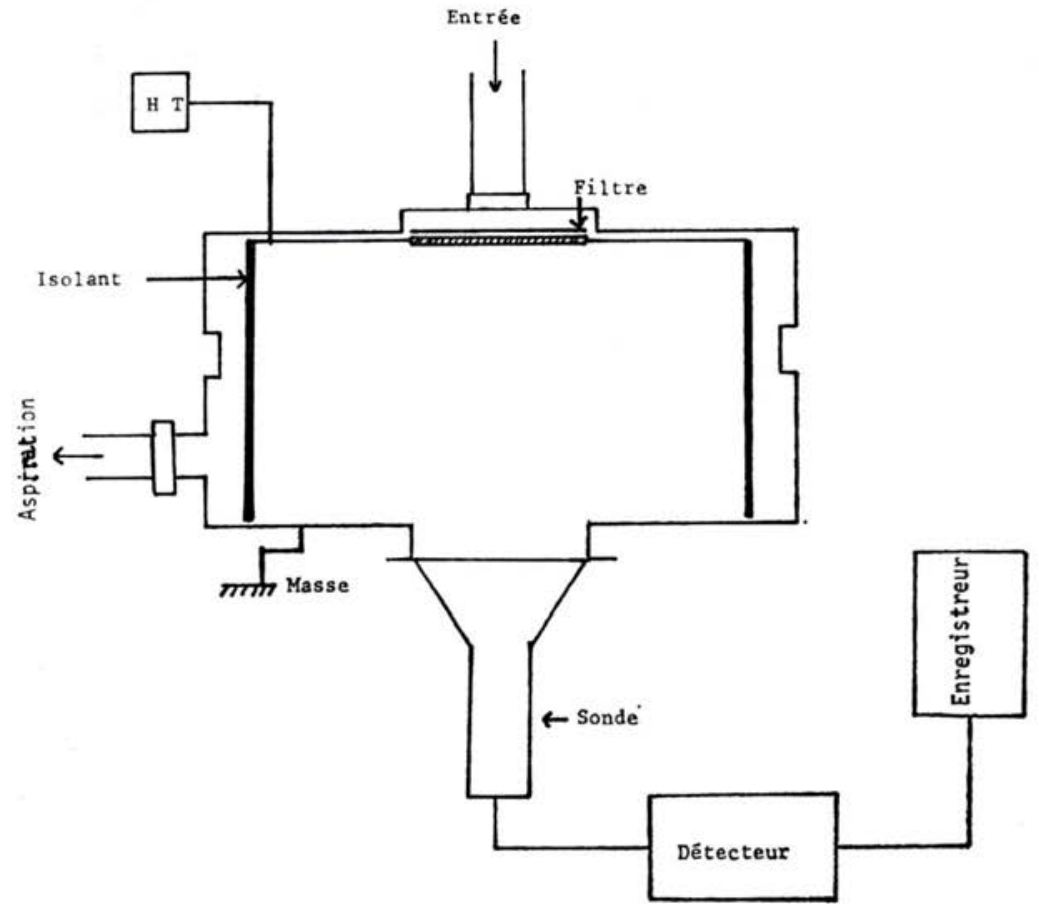

FIG. 2. - Mesure du radon en continu.

\section{c) Mesures}

Nous avons étudié ce dispositif avec notre enceinte sphérique en plexiglas remplie d'air fortement chargé en radon. De nombreuses expériences ont été nécessaires pour repérer la tension négative à partir de laquelle tout le radium $\mathrm{A}$ est piégé. On a fait varier cette tension négative à partir de 3 à $4000 \mathrm{~V}$ jusqu'à quelques dizaines de volts en valeur absolue. Pour chacune des valeurs de cette tension, nous avons observé l'allure des enregistrements. On constate, au bout de 1 à 2 min, l'apparition d'un palier de comptage. Mais ici, comme dans la méthode des deux filtres, l'importance de la filtration à l'entrée de la chambre est primordiale. Avec un seul filtre rose à haute

vol. $10-\mathrm{N}^{0} 1$ 


\section{TABLEAU I}

VALEURS DE $\boldsymbol{Z}$.

$$
\begin{aligned}
& Z=4,504 A F+164,69 B G-93,31 C H \\
& A=\mathrm{e}^{-\lambda_{2} T_{1}}-\mathrm{e}^{-\lambda_{2} T_{2}} \\
& B=\mathrm{e}^{-\lambda_{3} T_{1}}-\mathrm{e}^{-\lambda_{3} T_{2}} \\
& C=\mathrm{e}^{-\lambda_{4} T_{1}-\mathrm{e}^{-\lambda_{4} T_{2}}} \\
& F=1-\mathrm{e}^{-\lambda_{2} \mathrm{t}} \\
& G=1-\mathrm{e}^{-\lambda_{3} \mathrm{t}} \\
& \lambda_{2}=0,22726 \mathrm{~min}^{-1} \\
& \lambda_{3}=0,025864 \mathrm{~min}^{-1} \\
& \lambda_{4}=0,035185 \cdot \mathrm{min}^{-1} \\
& H=1-\mathrm{e}^{-\lambda_{4} t}
\end{aligned}
$$

$\begin{array}{cccc}\begin{array}{c}\mathrm{T}_{1} \\ (\min )\end{array} & \begin{array}{c}\mathrm{T}_{2} \\ (\mathrm{~min})\end{array} & \begin{array}{c}\mathrm{t} \\ (\min )\end{array} & \begin{array}{c}\mathrm{Z} \\ (\min )\end{array} \\ 1 & 6 & 1 & 0,49 \\ = & - & 2 & 0,884 \\ = & - & 4 & 1,46 \\ = & - & 10 & 2,312 \\ = & - & 15 & 2,656 \\ - & - & 5 & 2,597 \\ & & 10 & 3,803\end{array}$

efficacité à l'entrée, on constate une variation de palier à chaque variation de tension, ce qui prouve que la filtration est mauvaise.

On élimine ce phénomène par l'emploi d'un second filtre d'entrée. Il est aussi indispensable que la fixation de la chambre sur la sonde soit extrêmement étanche.

Ces conditions d'étanchéité et de filtration étant réalisées, on a pu montrer que le dépôt actif est piégé à une tension inférieure à $500 \mathrm{~V}$ en valeur absolue, ce qui a déjà été constaté dans les mines d'uranium. La vitesse d'apparition du palier ne varie pas sensiblement en fonction de la haute tension (elle est de l'ordre de 1 à $3 \mathrm{~min}$ ) et son existence permet de supposer qu'il n'y a pas de pollution sensible de la sonde par l'accumulation de dépôt actif. L'activité $\alpha$ comptée serait donc due à un flux statistiquement constant émis en direction de la sonde. Nous pensons que ce flux provient en quasi-totalité de la désintégration $\alpha$ du radon, mais il s'y ajoute certainement les $\alpha$ émis par les particules non piégées, en particulier les atomes de radium A neutres. L'un d'entre nous [12] a montré, en effet, que la proportion de ces atomes peut atteindre $20 \mathrm{p}$. cent de la totalité du radium $\mathrm{A}$ à l'origine.

Nous avons pu confirmer qu'il n'y a pas de pollution apparente de la sonde en modifiant la polarité de la plaque collectrice par l'application d'une tension positive de $1000 \mathrm{~V}$. Les ions radium A formés sont alors attirés vers le bas, une partie d'entre eux atteint la sonde et s'y dépose. On assiste aussitôt 
à une remontée brusque de l'activité ( fig. 3 : partie $A B$ ). Si l'on revient de nouveau à une tension négative, on observe une nouvelle décroissance assez rapide durant les premières minutes où l'activité du radium $\mathrm{A}$ capté par la sonde est prédominante (partie $C D$ ) et plus lente lors de la décroissance du radium $C^{\prime}$ seul (partie $D E$ ). L'état de départ est de nouveau atteint après plusieurs heures $(6$ ou $7 \mathrm{~h})$ ce qui correspond à la disparition totale du dépôt piégé par la sonde.

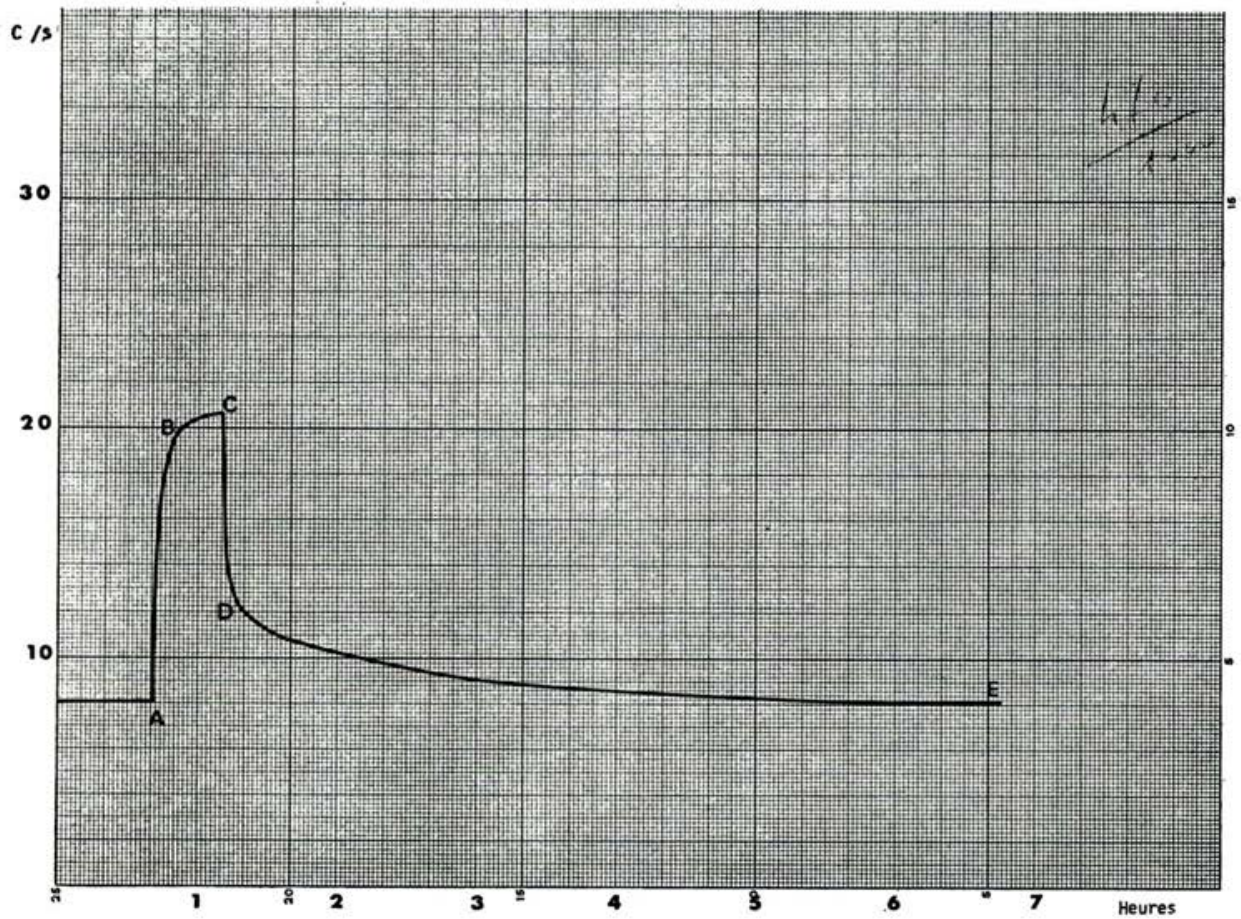

Fig. 3. - Activité de la plaque collectrice en fonction de la tension.

Compte tenu de nos essais, nous adoptons une tension négative de $-1000 \mathrm{~V}$.

L'étalonnage de la sonde avec les fioles scintillantes est tel que 1 coup/s correspond à $1000 \mathrm{pCi} / \mathrm{l}$. La courbe de la figure 4 montre que la relation entre les indications du DSM et l'activité en picocuries par litre est linéaire. Ce résultat a déjà été obtenu par DuPorT [13]. La sensibilité du DSM est de 0,1 coup/s, soit une sensibilité de la chambre de $100 \mathrm{pCi} / 1$.

La possibilité d'enregistrer de façon continue la variation de concentration en radon présente un très grand intérêt dans les mines d'uranium, dans le cadre de la protection des mineurs, moyennant quelques modifications

vol. $10-\mathrm{N}^{\circ} 1$ 
de la sonde afin d'améliorer son rendement, et de la chambre pour palier une hygrométrie élevée pouvant entraîner des phénomènes de conduction

$c_{R_{n} \cdot(100 \cdot p a / l)}$

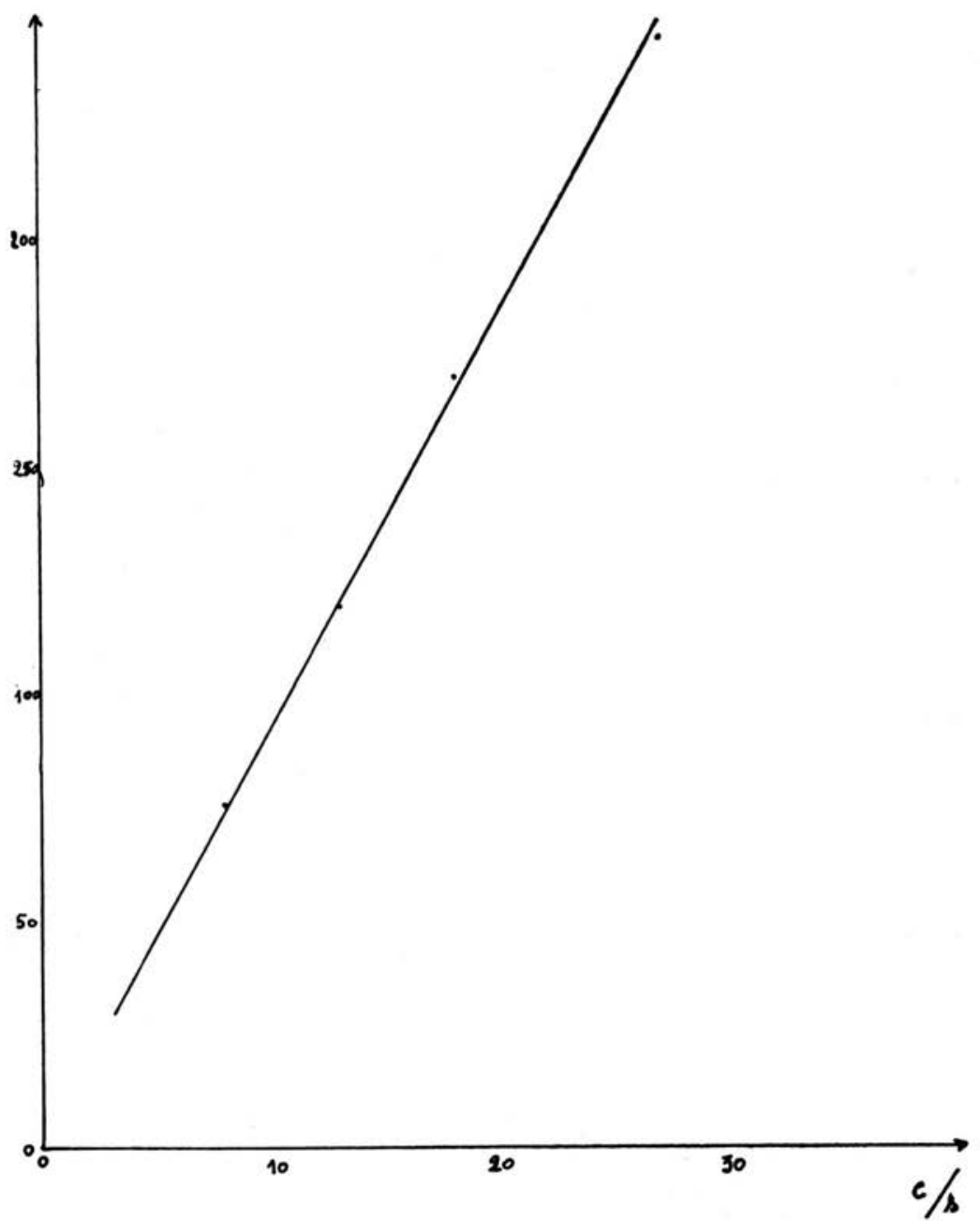

Fig. 4. - Relation entre les indications du DSM (en coups/s) et la concentration en radon.

des parties isolantes. L'utilisation du silicagel pour dessécher l'air représente une solution qui peut sembler efficace, mais sa saturation limite la durée de l'expérience. 


\section{CONCLUSION}

Les trois méthodes que nous venons d'étudier sont toutes trois utilisables pour la mesure directe de la teneur en radon de l'air d'une mine d'uranium. La troisième présente l'avantage d'un enregistrement continu, mais l'utilisation des hautes tensions et la présence d'isolants rend son utilisation délicate dans l'air humide d'une mine. De plus, elle est actuellement moins sensible que les autres.

Finalement, il nous semble que les deux premières méthodes sont celles qui semblent les mieux adaptées à ce type de problème. Celle des fioles a l'inconvénient de nécessiter une attente de $3 \mathrm{~h}$ mais ne demande aucun dispositif de pompage, contrairement à celle des deux filtres qui, elle, donne des résultats plus rapides.

Ce travail a pu être effectué grâce à un contrat entre le laboratoire de Physique des Aérosols et de Radioactivité atmosphérique de la Faculté des Sciences de Brest (Prof. Renoux), et le Service technique d'Études de Protection et de Pollution atmosphérique du C. E. A. (MM. Pradel et Zetrwoog). Nous remercions MM. François, Duport et Blondeau qui nous ont aidés lors de nos expériences dans les mines d'uranium.

\section{BIBLIOGRAPHIE}

[1] Billard F. et Pradel J. Le thoron et les risques associés dans la manipulation des composés du thorium. 2. Conférence int. sur l'utilisation pacifique de l'énergie atomique, Genève, juin 1958, Genève, Nations Unies, 1959, P/338.

[2] Israel $\mathbf{H}$. et Israel G. W. A new method of continuous measurements of radon (Rn-222) and thoron (Rn-220) in the atmosphere. Tellus, 1966, 18, 557-561.

[3] Fontan J. Le dosage des radioéléments gazeux donnant des produits radioactifs de filiation. Application à la mesure de la radioactivité naturelle de l'atmosphère. Thèse, Doctorat ès Sciences, Toulouse, 1964.

[4] Newstein H., Cohen L. D. et Krablin R. An automated atmospheric radon sampling system. Atmos. Environ., 1971, 5, 823-831.

[5] Thomas J. W. et Le Clare P. C. A study of the two-filter method for radon 222. Hlth Phys., 1970, 18, 113-122.

[6] TAN C. W. Diffusion of desintegration products of inert gases in cylindrical tubes. Int. J. Heat Mass. Trans., 1969, 12, 471.

[7] Bricard J., Girod P. et Pradel J. Spectre de mobilité des petits ions radioactifs de l'air. C. R. Acad. Sci., 1965, 260, 6587-6590.

[8] Strong J. C. et Duggan M. J. Ann. occup. Hyg., 1973, 16, 27-31.

voL. $10-\mathrm{N}^{\circ} 1$ 
[9] Billard F., Chevalier G. et Pradel J. Étude de matériaux filtrants et d'appareils de protection à l'aide d'aérosols radioactifs. 5 . Colloque int. sur les poussières, Paris, mars 1959 et Rapport CEA-1524, 1960.

[10] Parnianpour H. Étude de la pénétration des aérosols dans les couches filtrantes, Thèse, Doctorat ès Sciences et Rapport CEA-R 3347, 1967.

[11] Tymen G. et Renoux A. Bull. Soc. Scient. de Brest, juillet 1974.

[12] Renoux A. Étude des ions radioactifs de l'atmosphère. Thèse, Doctorat ès Sciences, Paris 1965 et Rapport CEA-R-2771.

[13] Duport P. et Madelaine G., Communication personnelle, 1973. 